\title{
Nox2 Is Required for Macrophage Chemotaxis towards CSF-1
}

\author{
Sanjay Chaubey ${ }^{1}$, Gareth E. Jones ${ }^{2}$, Ajay M. Shah ${ }^{1}$, Alison C. Cave ${ }^{1}$, Claire M. Wells ${ }^{3 *}$
}

1 Cardiovascular Division, The James Black Centre, King's College London BHF Centre of Excellence, London, United Kingdom, 2 Randall Division of Cell and Molecular Biophysics, King's College London, London, United Kingdom, 3 Division of Cancer Studies, King's College London, London, United Kingdom

\begin{abstract}
Macrophage migration and infiltration is an important first step in many pathophysiological processes, in particular inflammatory diseases. Redox modulation of the migratory signalling processes has been reported in endothelial cells, vascular smooth muscle cells and fibroblasts. However the redox modulation of the migratory process in macrophages and in particular that from the NADPH oxidase-2 (Nox2) dependent ROS has not been established. To investigate the potential role of Nox2 in the migratory response of macrophages, bone marrow derived macrophages were obtained from WT and NOX2 knockout mice (Nox2KO) and subjected to CSF-1 stimulation. We report here that loss of Nox2 expression in BMM resulted in a significant reduction in the CSF-1 induced spreading response suggesting that Nox 2 can modulate cytoskeletal events. Moreover, Nox2KO BMMs were deficient in cellular displacement in the presence of CSF-1. More significantly, when challenged with a gradient of CSF-1, Nox2KO BMMs showed a complete loss of chemotaxis accompanied by a reduction in cell migration speed and directional migration persistence. These results point to a specific role for Nox2KO downstream of CSF-1 during the BMM migratory response. Indeed, we have further found that Nox2KO BMMs display a significant reduction in the levels of ERK1/2 phosphorylation following stimulation with CSF-1.Thus Nox2 is important in BMM cellular motion to CSF-1 stimulation and necessary for their directed migration towards a CSF-1 gradient, highlighting Nox2 dependent signalling as a potential anti-inflammatory target.
\end{abstract}

Citation: Chaubey S, Jones GE, Shah AM, Cave AC, Wells CM (2013) Nox2 Is Required for Macrophage Chemotaxis towards CSF-1. PLoS ONE 8(2): e54869. doi:10.1371/journal.pone.0054869

Editor: Yick-Pang Ching, The University of Hong Kong, Hong Kong

Received September 5, 2012; Accepted December 17, 2012; Published February 1, 2013

Copyright: @ 2013 Chaubey et al. This is an open-access article distributed under the terms of the Creative Commons Attribution License, which permits unrestricted use, distribution, and reproduction in any medium, provided the original author and source are credited.

Funding: SC, AC and AMS were supported by the British Heart Foundation. CW is supported by the Guy's and St. Thomas Charity. The authors also acknowledge support from the Department of Health via a National Institute for Health Research (NIHR) Biomedical Research Centre award to Guy's \& St Thomas' NHS Foundation Trust in partnership with King's College London and King's College Hospital NHS Foundation Trust. The funders had no role in study design, data collection and analysis, decision to publish, or preparation of the manuscript.

Competing Interests: The authors have declared that no competing interests exist.

* E-mail: claire.wells@kcl.ac.uk

\section{Introduction}

Several isoforms of the gp91 $1^{\text {phox }}$ catalytic subunit of NADPH oxidase have been described. These isoforms are now termed NOXs, and comprise Nox1-5, Duox1 and 2 with Nox2 being the new name for gp9 $1^{\text {phox }}[1]$. Superoxide-generating enzymes are a major sources of ROS and have been shown, by way of redox modulation of cellular signalling, to play important roles in disease pathophysiology, in particular inflammatory diseases [2,3,4].

The progression of atherosclerosis is an inflammatory process requiring cellular migration and infiltration. Indeed, it has been shown that within atherosclerotic plaques, in $\mathrm{ApoE}^{-/-}$mice, macrophages were a prominent source of Nox2 [5]. Furthermore, the Nox2 expression was elevated before the appearance of lesions, consistent with a causal role for the enzyme in the early activation of critical pro-atherogenic pathways. Importantly, global deletion of Nox2 in the $\mathrm{ApoE}^{-/-}$mice inhibited atherosclerotic lesion development in the aortic arch, thoracic and abdominal aorta [5].

In keeping with atherosclerosis, a high cholesterol diet which is implicated in this process, has been shown to induce an inflammatory response in the post capillary venules [6]. This hypercholesterolemia induced inflammatory response was demonstrated to be dependent on superoxide production, in particular that from NADPH oxidase. Thus NADPH oxidase superoxide production is a critical event that initiates the leukocyte endothelial cell adhesion in postcapillary venules in mice following a high cholesterol diet [6].

Interestingly there is growing evidence in the literature for a role of the Nox family proteins in modulating the processes involved in cellular migration. For example, Rac stimulates actin polymerisation by several mechanisms including NADPH oxidase mediated ROS production [7]. The dephosphorylation of the cytoskeletal regulator cofilin following PDGF stimulation has also been shown to be Noxl dependent $[8,9]$. During fibronectin/integrin mediated cell adhesion, ROS is dramatically increased by Rac-1 dependent activation of NADPH oxidase [10]. Recently Nox4 has also been shown to be a key player in the regulation of stress fibre formation and focal adhesion turnover in VSMC [11]. NADPH generated ROS has also been shown to be important in invadopodia formation facilitating the invasive behaviour of cancer cells [12].

In keeping with the regulatory role of Nox2 in cellular migration, Rac1- and Nox2-dependent NADPH oxidase have been shown to play an important role in endothelial cell migration, as seen during tissue repair in response to injury, angiogenesis, and wound healing $[13,14,15]$. Also oxidised LDL, which extensively accumulates in atherosclerotic plaques, can stimulate ROS production in macrophages through $\mathrm{NADPH}$ 
oxidase, which stimulates downstream expression of proinflammatory cytokines. [16]. These cytokines have been shown to stimulate smooth muscle cell migration important in the progression of atherosclerotic plaques. However the direct role of Nox2 in the migration of macrophages, important in pathophysiological processes such as atherosclerosis and inflammatory diseases, has not been well established. This paper investigates whether the Nox2-dependent NADPH oxidase modulates the migration of macrophages and in particular to a common tissue chemoattractant, CSF-1.

\section{Materials and Methods}

\section{Reagents}

All chemicals and DMEM were purchased from Sigma. CSF-1 was purchased from RandD systems, USA. Versene for cell detachment was purchased from Gibco. Phalloidin-FITC was purchased from Sigma. Antibodies to phospho and total ERK1/2 and Akt were purchased from Cell Signalling Technology.

\section{Animal Husbandry and Maintenance}

All mice were maintained in a designated facility in accordance with the Code of Practice for the Housing and Care of Animals Used in Scientific Procedures. Mice were housed up to a maximum of 5 per cage and had free access to water and normal food chow. The mice were anaesthetised using Isoflurane $(2-2.5 \%$ isoflurane/oxygen). Once deep anaesthesia had been reached the mice were terminally culled by cervical dislocation. All experimental procedures were carried out under the authority of a Home Office Personal Licence and Project Licence. All animal procedures were performed following in accordance with the Guidance on the Operation of the Animals (Scientific Procedures) Act, 1986 (UK Home Office) and approved by the King's College London Animal Care and Use Committee.

\section{Isolation and Culture of Mouse Primary Bone Marrow Derived Macrophages}

The murine femoral bones were harvested after the mice were culled using terminal anaesthesia. All the surrounding tissue on the bone was removed and the bone pierced at both ends with a 21-gauge needle. The bone marrow was flushed out of the bone with macrophage starve medium (RPMI 1640 with L-glutamine, $1 \%$ Essential amino acids, 1\% sodium pyruvate, $1 \% \mathrm{P}+\mathrm{S}, 10 \%$ FCS and $0.5 \% \quad \beta$ mercaptoethanol). Cells were then centrifuged and the pellet resuspended in macrophage starve medium. The cells were then counted and $2 \times 10^{5}$ cells/ $\mathrm{cm}^{2}$ seeded onto $10 \mathrm{~cm}$ petri dishes for 3 days in macrophage growth media (starve medium plus M-CSF1 at $30 \mathrm{ng} / \mathrm{ml}$ ). After 3 days the non-adherent population of cells containing the monocytes was removed. The cells were centrifuged, resuspended and counted. The cells were then seeded onto $6-\mathrm{cm}$ bacterial culture plates at a density of $10^{5}$ cells $/ \mathrm{mL}$. The cells are incubated for a further 5 days in the presence of M-CSF-1 as above. The differentiated BMMs become adherent and were harvested on day 5 for experimentation.

\section{Time-lapse Microscopy and Migration Analysis}

To study random cell motion, cells were seeded onto 6 well plastic petri dishes at a density of $2 \times 10^{4}$ cells $/ \mathrm{ml}$ in macrophage growth medium and incubated overnight. Following incubation, cells were starved of CSF-1 in macrophage starve medium for 8 hours. The cells were then stimulated with CSF-1 by the reintroduction of CSF-1 (30 ng/ml) containing growth media. Cell images were collected using a Pulnix CCGD camera, taking a frame every $5 \mathrm{~min}$ for $18 \mathrm{~h}$ using AQM acquisition software (Andor, UK). Subsequently all the acquired time-lapse sequences were displayed as a movie and each cell in the first frame was tracked for the whole of the time-lapse sequence, using Motion Analysis software (Andor, UK) This resulted in the generation of a sequence of position co-ordinates relating to each cell in each frame. All the tracks were centred to co-ordinate $(0,0)$ to better view the distance travelled. A circular horizon distance was set and the number of cells from the total population that reached the horizon distance was monitored. The random speed and the persistence in the random motion was calculated and compared. Mathematical analysis was carried out using Mathematica 6.0TM workbooks [17]. P-values less 0.05 were accepted as statistically significant.

To study chemotaxis, cells were seeded on acid washed coverslips at a density of $2 \times 10^{4}$ cells $/ \mathrm{ml}$ in macrophage growth medium and incubated overnight. Following incubation cells were starved of CSF-1 in macrophage starve medium for 8 hours. The coverslips were then mounted onto Dunn chemotaxis chambers as previously described [18] using recombinant murine CSF-1 $(30 \mathrm{ng} / \mathrm{ml})$ as the chemoattractant. Cell images were collected and analysed as described above.

\section{Immunofluoresence}

BMMs were seeded on glass coverslips at $2 \times 10^{5}$ cells per coverslip and maintained in macrophage growth or macrophage starve medium followed by CSF-1 stimulation as indicated. Cells were washed with PBS, fixed with $4 \%$ paraformaldehyde permeabilised and stained for actin using phalloidin-FITC. The actin images were collected on IX71 Olympus microscope and cell images were analysed using ImageJ.

\section{Immunoblotting}

Cells were seeded onto 6 well plates and maintained or CSF-1 deprived as outlined above. Following stimulation cells were lysed as previously described [17] and lysates subjected to acrylamide gel electrophoresis as previously described [17]. Protein membranes were blocked and probed with primary and secondary antibodies as indicated. The blots were developed using Pierce ${ }^{\circledR}$ ECL Western Blotting Substrate Kit (Thermo Scientific). The autoradiograph were scanned and band densities were quantified with Kinetic Imaging software to obtain the ratio of phosphorylated protein to total protein.

\section{Results}

\section{Nox2KO Macrophages have an Increased Spread Area}

We were interested in establishing whether Nox2 plays a role in the infiltration of macrophages at sites of inflammatory response, such as those that are thought to be associated with tissue repair or conditions such as atherosclerosis. Many of the signalling pathways that regulate cellular migration are the same as those controlling cellular morphology. Therefore we first analysed the cell morphology of WT and macrophages derived from Nox2 knockout mice (Nox2KO) under basal growing conditions. We observed no difference in the global actin architecture between WT and Nox2KO BMMs but did find that Nox2KO BMM had a larger spread area and were reproducibly more elongated compared to WT BMM (Figure 1a and b). CSF-1 is well known to stimulate cell morphology changes in BMMS [17], where deprivation of CSF1 induces cell elongation and re-stimulation leads to centrifugal spreading [17]. Therefore we next tested whether Nox2 expression was required for a morphological response to CSF- 
1. Interestingly, following CSF-1 deprivation, WT and Nox2KO macrophages reduced their area to a similar size (Figure 2). Moreover, CSF-1 stimulation induced cell spreading in both WT and Nox2KO BMMs (Figure 2). However whilst, WT macrophages exhibited an increase of $79 \%$ in spread area compared to their spread area under starved conditions Nox2KO macrophages only exhibited a $55 \%$ increase after 5 minutes of CSF-1 stimulation. Although not statistically significant, taken together with the reduced cell area for growing cells, these data do suggest that Nox2 expression is in part required for normal BMM behaviour. Interestingly, both populations were able to centrifugally spread in response to CSF-1 (Figure 2), suggesting that CSF-1 responses are not completely dependent on Nox2 in BMMs.

\section{Nox $2^{-/-}$BMM Showed Reduced Random Motion Following CSF-1 Stimulation}

Given that we had observed a change in growing cell spread area and a reduction in the spread area in response to CSF-1, we speculated that Nox2KO BMMs might have defects in CSF1 simulated migration. Initially we tested random migration and found that there was a small but reproducible reduction in cell migration speed observed in the Nox $2 \mathrm{KO}$ population. In the presence of CSF-1 WT cells exhibited a mean migration speed of $0.71 \mu \mathrm{m} / \mathrm{min}$ whilst Nox2KO BMMs exhibited a mean migration speed of $0.67 \mu \mathrm{m} / \mathrm{min}$ (Figure 3E). Moreover we found a significant reduction $(\mathrm{p}=0.02)$, in cell displacement (Figure 3C and D). Where a reduced number of the Nox2KO BMMs population were able to reach the set horizon compared to WT BMMs. We speculate that this may be due in part to the slightly reduced cell speed but could also be attributed to the observation that Nox2KO BMMs tended to oscillate in movement more than WT BMMs and therefore not achieve overall displacement, this is partly reflected in the increased persistence of migration (Figure 3F) we recorded for the Nox2KO BMMs. These results suggest that Nox2 does play a role in the migration of BMMs following CSF-1 stimulation.

\section{A}
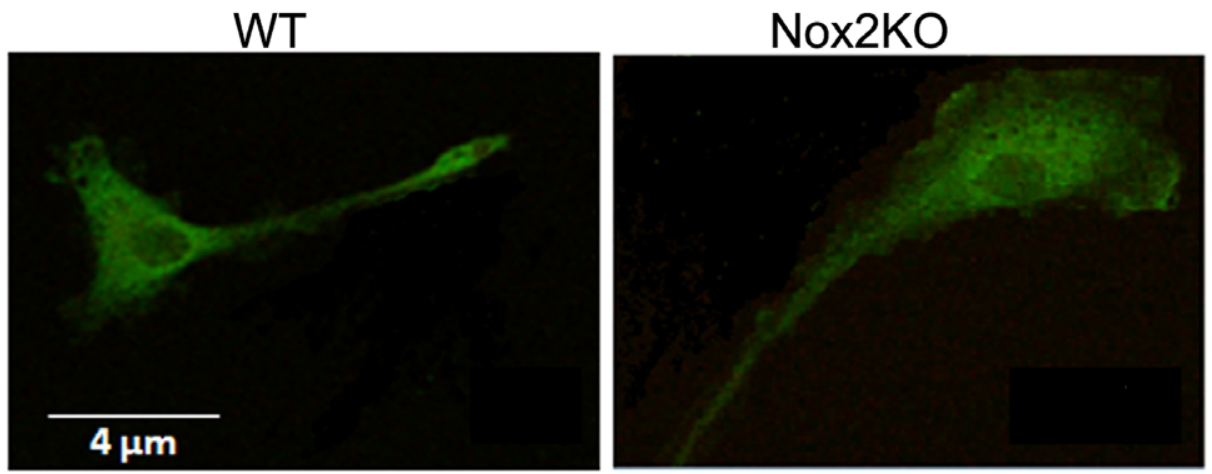

B
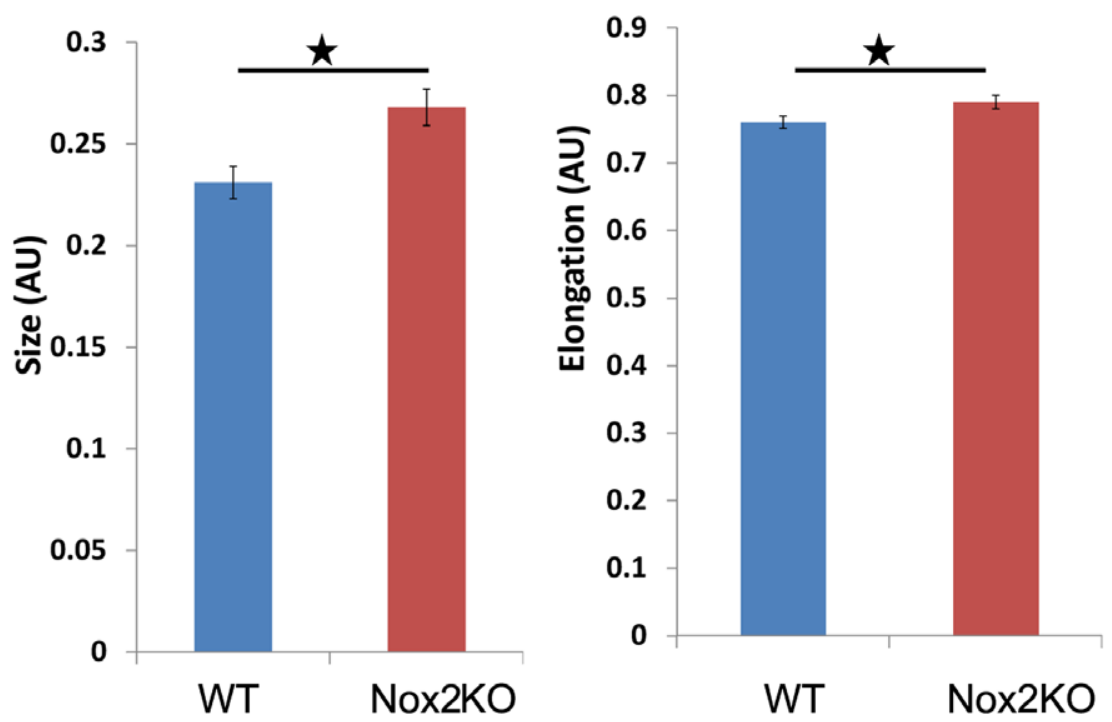

Figure 1. Nox2KO BMMs have increased cell area. a) WT and Nox2KO BMMs were fixed and stained for F-actin. b) Cell area and cell elongation analysis was conducted using ImageJ software. Representative of three independent experiments with $>30$ cells measured for each experiment. ${ }^{*}=p<0.05$.

doi:10.1371/journal.pone.0054869.g001 

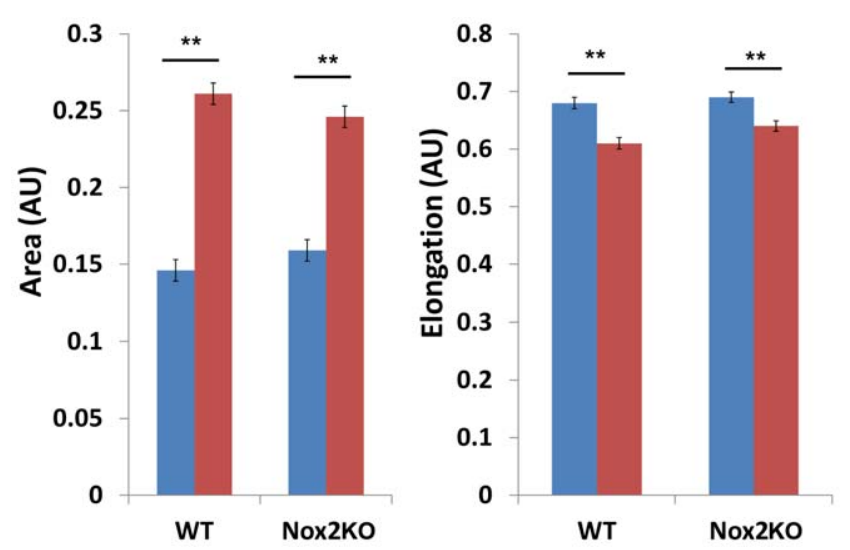

Figure 2. BMMs respond to CSF-1 stimulation. Cells were seeded on coverslips, CSF-1 deprived then re-stimulated with CSF-1 for 5 minutes. Cells were fixed and stained for F-actin and cell area and cell elongation analysis was conducted using ImageJ software. Representative of three independent experiments with $>30$ cells measured for each experiment. Where blue bars represent CSF-1 starved and red bars represent CSF-1 stimulated cells $* *=p<0.005$.

doi:10.1371/journal.pone.0054869.g002

\section{Nox2 $2^{-/-}$Cells did not Exhibit a Chemotactic Response Towards CSF-1}

BMM are known to have a chemotactic response to CSF-1 [17], and in a physiological context are likely to be responding to a gradient of chemoattractant rather than global stimulation. Thus we next challenged the WT and Nox2KO BMMs to chemotax towards a source of CSF-1 using the Dunn Chemotaxis Chamber. Whilst WT BMM were able to efficiently chemotax towards CSF1, Nox2KO BMMs completely lost their chemotactic response (Figure 4). Loss of chemotaxis can sometimes be attributed to a reduction in cell speed and we did indeed find that there was a significant reduction in mean cell migration speed in the Nox $2 \mathrm{KO}$ population $(\mathrm{p}<0.001)$ (Figure $\mathbf{4 G}$ ). However, we found that cell persistence was also significantly $(\mathrm{p}<0.001)$ reduced in Nox2KO BMM (Figure 4D) as compared to WT suggesting that Nox2KO cells were unable to respond to the CSF-1 gradient. This would suggest a more significant role for Nox2 in the directed migration of the BMMs compared to random migration.

\section{Nox $2^{-1-}$ Macrophages have an Attenuated Signalling Response to CSF-1}

Given that we have detected changes in both cellular morphology, cell spreading and directed cell migration we reasoned that signalling downstream of CSF-1 may be altered in Nox2KO cells. CSF-1 is well known to stimulate both ERK [19] and Akt phosphorylation [20] in BMMs. Indeed, levels of ERK phosphorylation have been linked to cell spreading [19]. We found no difference in Akt phosphorylation downstream of CSF-1, however, Nox2KO BMMs have an attenuated phospho-ERK response (Figure 5). Where levels of ERK phosphorylation were significantly reduced following 15 mins of CSF-1 stimulation. Thus, Nox2KO BMM do have attenuated signalling downstream of CSF-1 stimulation.

\section{Discussion}

The coordination and synergy between the cytoskeletal dynamics at the leading edge, the strengthening of adhesion to the ECM and cellular contractility play a key role in the dynamics of cellular morphology and migration [21]. Redox signalling has been shown to be influential in this process at many different stages. In this paper Nox2 has been shown to play a role in regulating cellular morphology, random cellular motion and also to be critical in directed cellular migration, speed and chemotaxis. A key finding in this paper was that Nox2 in BMM was found to be important in random cellular motion and necessary in directed cellular motion.

WT and Nox2KO BMMs are morphologically different and this is reflected in the difference in mean spread area of these cells and the elongated shape of Nox2KO BMMs. However, both populations were able to respond to CSF-1. Although, the Nox2KO BMMs tended towards a slower response in CSF-1 induced cell spreading. The loss of Nox2 did result in a significant reduction in the random motility of $\mathrm{BMM}$ as observed by the lower numbers of BMM migrating to the set horizon following CSF-1 stimulation. Also the Nox2KO BMM showed more intrinsic persistence in their random movement. Random motion allows cells to explore their environment. The increased intrinsic persistence suggested the loss of Nox2 had resulted in a reduction in the cells ability to turn and explore their environment. Pankov et al [22] demonstrated that total Racl activity was important in determining whether random cell migration followed a more intrinsic random or directionally persistent pattern of motion. The data here suggests that, at least in part, some of these regulatory functions of Racl could be through Nox2.

In contrast to random motion, directional migration moves cells rapidly between points. When challenged with a gradient, loss of Nox2 in BMM resulted in a complete loss of chemotaxis towards CSF-1 and a loss of cell migration and directional persistence. The BMM were able to sense and respond to CSF-1 stimulation as observed by the increase in the speed of WT and Nox2KO BMM, although Nox2KO BMMs were significantly slower than WT cells. Thus the loss of chemotaxis suggested a critical role for Nox2 further downstream from the cell sensing of the external signal and/or in cellular polarisation.

A possible mechanism by which Nox2 could affect the directionality of the cell could be by the redox modulation of the intracellular signalling gradients established by phophoinositides. The phosphoinositides $\operatorname{Ptd} \operatorname{Ins}(3,4,5) \mathrm{P}_{3} \quad\left(\mathrm{PIP}_{3}\right)$ and $\operatorname{PtdIns}(3,4) \mathrm{P}_{2}\left[\mathrm{PI}(3,4) \mathrm{P}_{2}\right]$ along with PI3K and PTENS are key signaling molecules in this process $[23,24]$. This process involves both localized accumulation and activation of PI3Ks, which generate $\mathrm{PIP}_{3} / \mathrm{PI}(3,4) \mathrm{P}_{2}$, and the phosphatase PTEN, which removes them [25]. Cells with altered PI3K or PTEN activity can usually show chemokinesis but exhibit a significantly reduced chemotaxis [24,26]. Many of these signaling molecules have been shown to be redox sensitive. Leslie et al [27] demonstrated that oxidative stress with $\mathrm{H}_{2} \mathrm{O}_{2}$ resulted in the inactivation of PTEN. PTEN is a member of the Protein Tyrosine Phosphatase family which can be physiologically regulated through reversible oxidation resulting in their inactivation $[28,29]$. The inactivation of PTEN results in an increase in cellular phosphoinositides and thus the loss of any gradient established by the phosphoinositides to a chemoattractant signal. Also phosphoinositides, $\operatorname{Ptd} \operatorname{Ins}(3,4,5) \mathrm{P}_{3}$ $\left(\mathrm{PIP}_{3}\right)$ and $\operatorname{Ptd} \operatorname{Ins}(3,4) \mathrm{P}_{2}\left[\mathrm{PI}(3,4) \mathrm{P}_{2}\right]$, have been shown, by way of their Phox domains for subunits $\mathrm{p} 40^{\text {phox }}$ and $\mathrm{p} 47^{\text {phox }}$, to be involved in the recruitment and activation of these Nox regulatory proteins, [30,31] thus establishing a means for the redox modulation of these downstream signalling molecules.

Cellular polarisation is equally important for directed cellular migration in which the small GTPase are important in this process and in particular Cdc42. Cdc42 is a master regulator of cell polarity by being active towards the front of migrating cells [32] and by restricting where lamellipodia forms [33]. Its importance is 
A

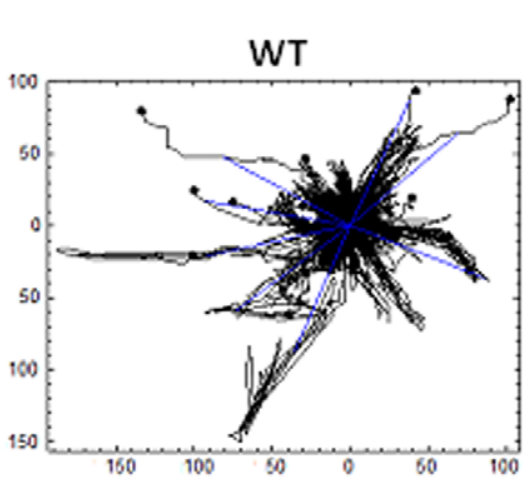

C

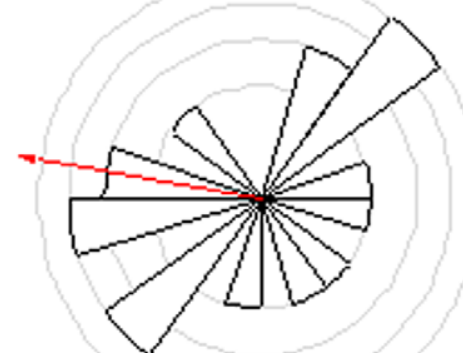

E

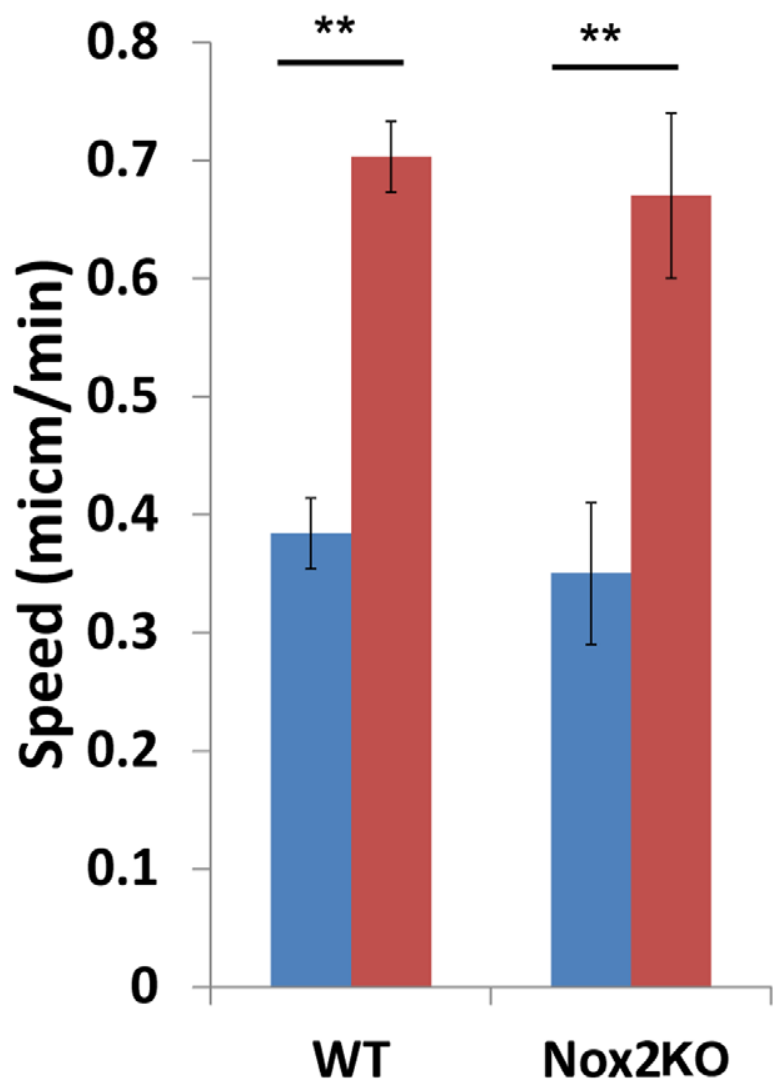

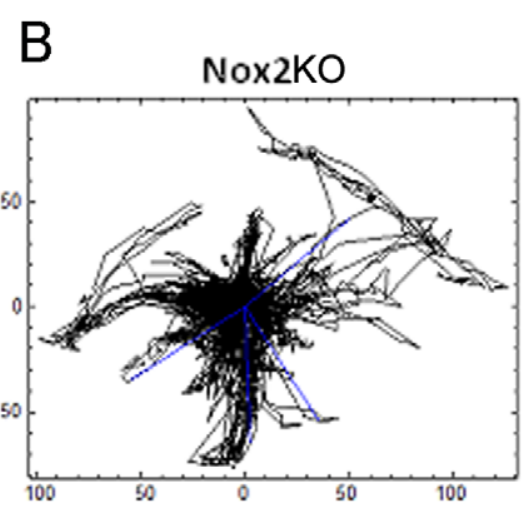

D

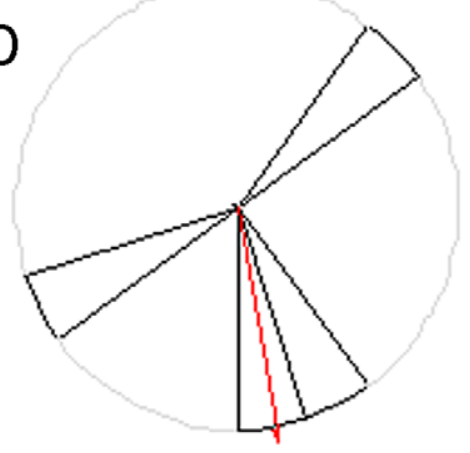

F

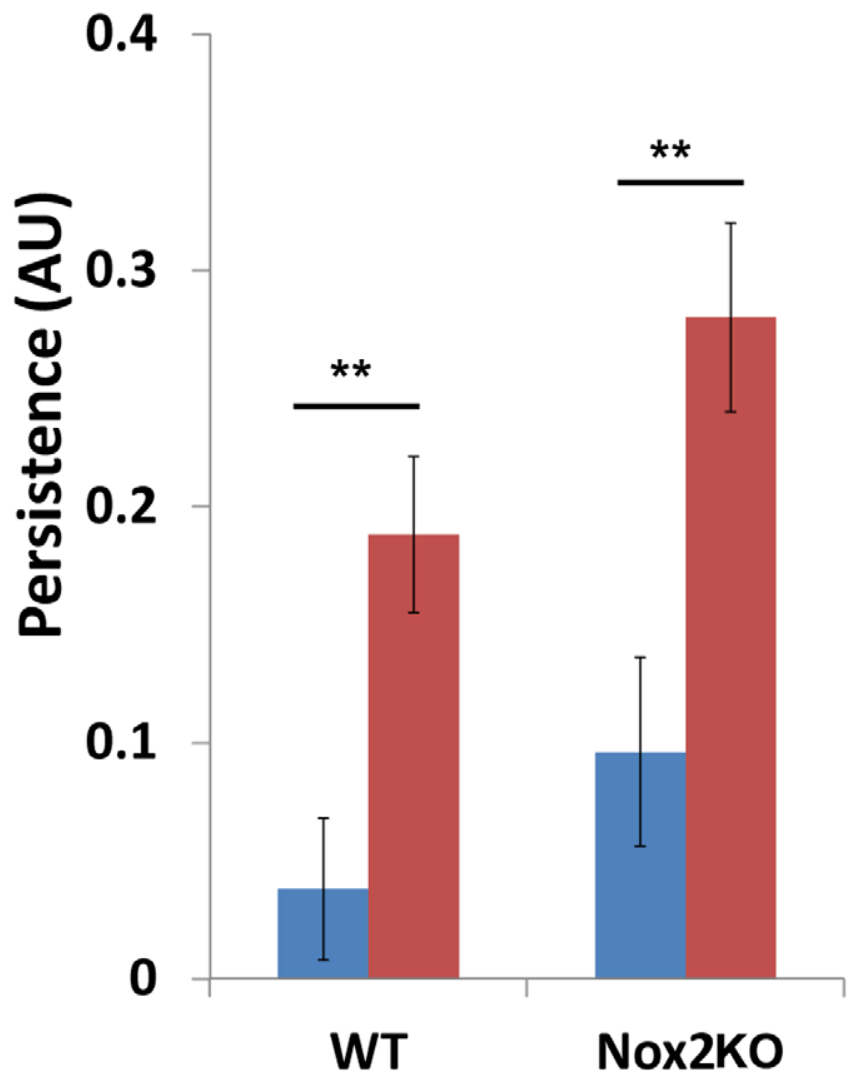


Figure 3. Nox2KO BMMs have reduced cell displacement. WT and Nox2KO BMMs were seeded on plastic, CSF-1 deprived then stimulated with CSF-1 and imaged as described in material and Methods. A and B) cell tracks of WT and Nox2KO BMM respectively. The tracks have been re-set to co-ordinate $(0,0)$. C and D) The number of cells reaching a set circular horizon was monitored and found to be significantly $(p=0.02)$ more in the WT than Nox2KO BMM following CSF-1 stimulation. (E and F) mean cell migration speed and mean persistence of direction were calculated from cell tracks above (see material and methods for details). Where blue bars represent CSF-1 starved and red bars represent CSF-1 stimulated cells $* *=p<0.005$.

doi:10.1371/journal.pone.0054869.g003

confirmed in experiments where both the inhibition and global activation of Cdc42 disrupts the directionality of migration $[18,34]$. How Cdc42 and Nox2 are associated is not entirely clear however evidence from the literature suggest that in an in-vitro cell free experiment Cdc42 can act as a competitive inhibitor of Rac-1 and Rac-2 activation of cytochrome $\mathrm{b}_{558}$ and therefore ROS production [35]. Cell polarisation is reflected in the ability of a cell to modulate its shape during CSF-1 deprivation and re-stimulation.

It was interesting to note that whilst mean cell migration speed was significantly reduced during directed migration such a large difference in effect was not observed during random migration in the Nox2KO BMMs following global CSF-1 stimulation. The molecular mechanism for the Nox2 dependency on the speed of BMM migration is not established, but many of the proteins involved in the control of actin cytoskeleton reorganisation are redox sensitive such as PTENS and PI3K [36]. Lamellipodia formation in moving cells requires cycles of actin polymerization and depolymerisation. Rac stimulates actin polymerization by several mechanisms, including NADPH oxidase mediated ROS production [7].The relation between the actin cytoskeleton and ROS seems to be bi-directional. Thus, cortactin, an actin-binding protein that has traditionally been found to regulate polymerization of the actin cortex, has also been shown to mediate $\mathrm{p} 47^{\text {phox }}$ translocation to the membrane during AngII induced activation of NADPH oxidase [37]. Moreover, actin activates Nox2 in neutrophils in a cell-free system, implying their direct effect on NADPH oxidase enzyme activity, and the destabilization of the actin cytoskeleton robustly enhances the neutrophil respiratory burst activity $[38,39]$. A more complete understanding of this bidirectional relation between NADPH oxidases and the actin cytoskeleton may shed further light on how it mediates migration.

The significantly reduced phosphorylation of ERK1/2 was in line with its important role in cellular migration and that of Nox2 in the activation of Ras/Raf/MEK/ERK signalling cascade downstream from the tyrosine receptors. ERK1/2 localise to the cell membrane [40] and to focal adhesions [41] and promote lamellipodium formation and spreading in epithelial cells [42]. Smith et al found that ERK $1 / 2$ activity was reduced in PAK $1^{-/}$ BMMs which displayed spreading defects compared with WT BMMs thus suggesting that optimal activation of ERK1/2 is required during BMM spreading. [19] We also found reduced activation of ERK1/2 in the Nox2KO BMM following CSF-1 stimulation suggesting a possible mechanism whereby Nox2 generated ROS is able to modulate the downstream response via activation of ERK.

Our data points to an involvement of NOX2 in BMM migration. It is interesting to note that different isoforms of

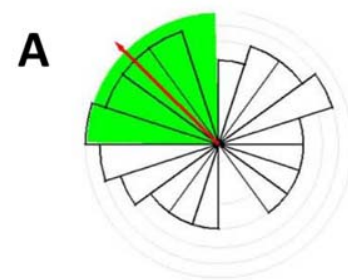

WT

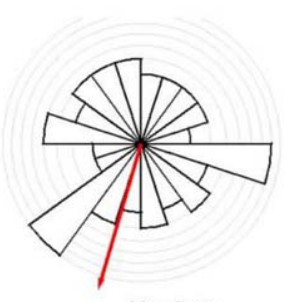

Nox2KO
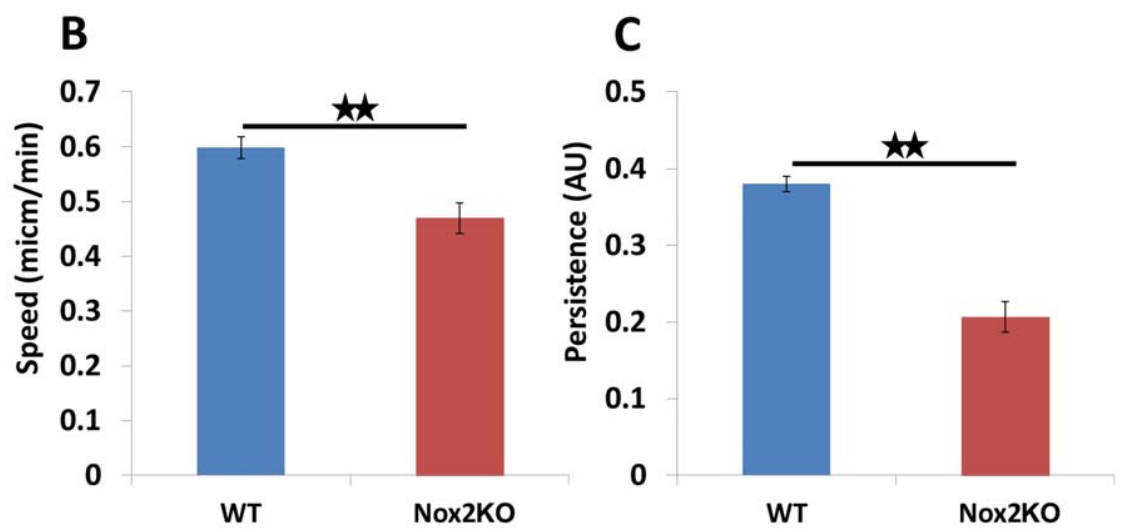

Figure 4. Nox2KO BMMs cannot chemotax towards a source of CSF-1. A) WT and Nox2KO BMMs were seeded on glass coverslips, deprived of CSF-1 and then placed in a gradient of CSF- 1 using the Dunn chemotaxis chamber. Cells were tracked and the tracks re-set to co-ordinate $(0,0)$ and represented by a circular histogram where the mean direction of cells is represented by a red arrow with $95 \%$ confidence interval (green wedge). Representative of three independent experiments. B and C) mean cell speed and mean persistence of direction were calculated from the tracks generated in $(A) .{ }^{* *}=p<0.001$.

doi:10.1371/journal.pone.0054869.g004 

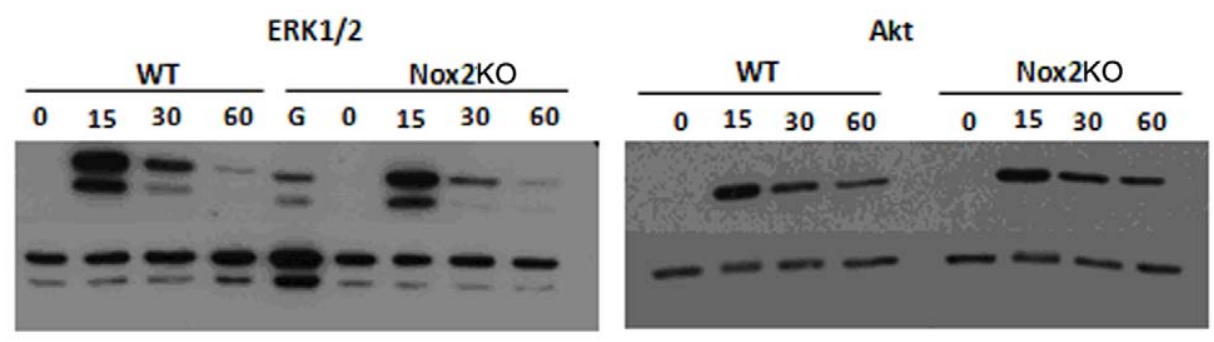

ERK1 phosphorylation

ERK2 phosphorylation

Akt phosphorylation

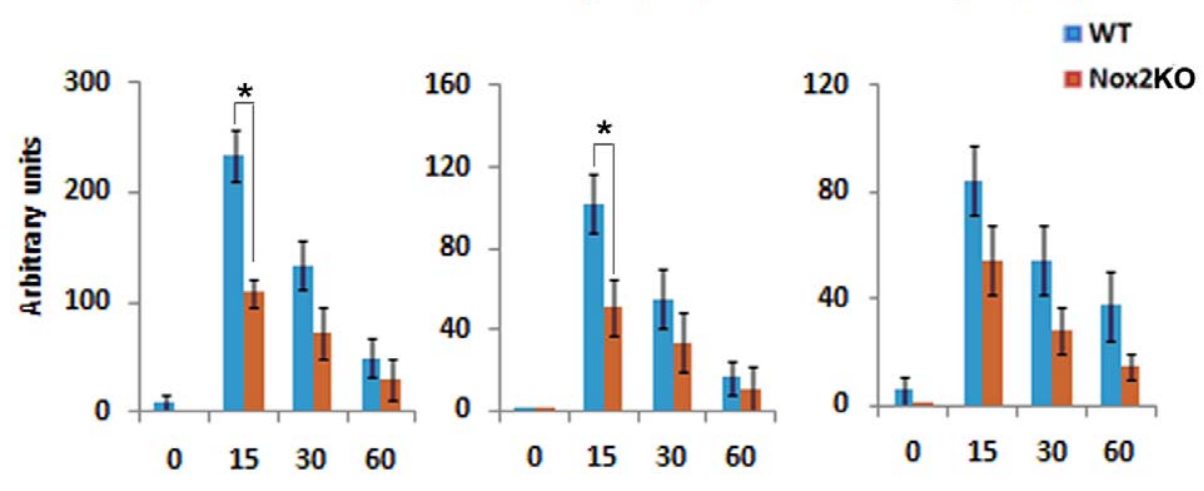

Figure 5. Nox2KO BMMs have reduced ERK phosphorylation downstream of CSF-1. A) WT and Nox2KO BMMs were CSF-1 deprived, then re-stimulated with CSF-1for the times indicated. Cells were lysed and probed for $\mathrm{pAKt}$, $\mathrm{pERK}$ and total protein.B) autoradiographs were analysed using AndorlQ and levels of pERK1, pERK2 and pAKT were normalised to loading controls. Data represents three independent experiments. ${ }^{*}=p<0.05$. doi:10.1371/journal.pone.0054869.g005

NADPH oxidase have also been shown to be involved in the migration of other cell types. Nox4 has also recently been found to be a key player in the regulation of stress fibre formation and focal adhesion turnover in VSMCs [11]. These findings suggest a potentially novel mechanism of local ROS production by which focal adhesion turnover is coordinated. Certainly a role of Nox2 in the regulation of such adhesion formation in BMM could explain the difference observed in their shape and then in their speed and persistence. Further studies of differences in the expression of integrins would increase the understanding of the exact underlying mechanism whereby the loss of Nox2 results in a reduction in the speed of migration in BMM. An important role for Noxl in the migration of VSMC to $\beta \mathrm{FGF}$ agonist stimulation has also been identified [43] in rat SMC where inhibition of Noxl significantly blocked migration.

In summary in order to initiate inflammation and tissue repair, the migration of macrophages into tissue is an important initial step. However the loss of Nox2 results in significant reduction in the random migration of BMM. On interrogating the BMM towards a directed target we have shown that the loss of Nox2 proved crucial as its loss resulted in the complete loss of chemotaxis. Nox2 was also important in the BMM speed and persistence towards a CSF-1 gradient with significant reductions in both. This loss of Nox2 also manifested itself in a reduced ERK1/ 2 phosphorylation and spreading responses to CSF-1 stimulation.

\section{Concluding Remarks}

We have investigated for the first time the role of Nox2 in macrophage migration. Data presented here indicates Nox2 expression is necessary in response to CSF-1 stimulated migration. This in-vitro behaviour could in part be related to in vivo phenotypes associated with Nox2. A complete deficiency of Nox2, as in patients with chronic granulomatous disease (CGD), is associated with hyperinflammation, suggesting that the normal functions of Nox2 in macrophages and potentially other inflammatory cells are essential in restricting or resolving inflammation. On the other hand, Nox2KO mice are protected against fibrosis that accompanies inflammatory repair processes in the liver $[44,45]$, heart $[46,47,48]$ and kidneys $[49,50]$. Furthermore, specific inhibition of Nox2 reduces macrophage infiltration into vessels in a model of angiotensin II-induced hypertension [51] whilst macrophages lacking Nox2 oxidase activity are reported to infiltrate less efficiently into atherosclerotic lesions [52] and the aorta [53]. No mechanisms to explain these observations were reported in these studies. Our current results suggest that Nox2dependent regulation of macrophage migration may underlie the effects on macrophage infiltration previously reported in experimental models of atherosclerosis and vascular disease. They further suggest that inhibition of Nox2 may be beneficial in such settings (all vascular disease) by inhibiting inflammatory infiltration. The development of novel therapeutics will however require a clear understanding of how this relationship is regulated.

\section{Author Contributions}

Conceived and designed the experiments: CMW GEJ AMS AC SC. Performed the experiments: SC. Analyzed the data: SC. Contributed reagents/materials/analysis tools: CMW GEJ. Wrote the paper: SG AMS CMW.

\section{References}

1. Lambeth JD (2004) NOX enzymes and the biology of reactive oxygen. Nat. Rev. Immunol. 4, 181-189.

2. Griendling KK, Sorescu D and Ushio-Fukai M (2000b) NAD(P)H oxidase: role in cardiovascular biology and disease. Circ. Res. 86, 494-501. 
3. Griendling KK, Sorescu D, Lassegue B and Ushio-Fukai M (2000a) Modulation of protein kinase activity and gene expression by reactive oxygen species and their role in vascular physiology and pathophysiology. Arterioscler. Thromb. Vasc. Biol. 20, 2175-2183.

4. Lassegue B, San MA, and Griendling KK (2012) Biochemistry, physiology, and pathophysiology of NADPH oxidases in the cardiovascular system. Circ. Res. 110, 1364-1390.

5. Judkins CP, Diep H, Broughton BR, Mast AE, Hooker EU, et al. (2010) Direct evidence of a role for Nox2 in superoxide production, reduced nitric oxide bioavailability, and early atherosclerotic plaque formation in $\mathrm{ApoE}-/-$ mice. Am. J. Physiol Heart Circ. Physiol 298, H24-H32.

6. Stokes KY, Clanton EC, Russell JM, Ross CR, and Granger DN (2001) $\mathrm{NAD}(\mathrm{P}) \mathrm{H}$ oxidase-derived superoxide mediates hypercholesterolemia-induced leukocyte-endothelial cell adhesion. Circ. Res. 88, 499-505.

7. Moldovan L, Moldovan NI, Sohn RH, Parikh SA, Goldschmidt-Clermont PJ (2000) Redox changes of cultured endothelial cells and actin dynamics. Circ. Res. 86, 549-557.

8. Lee MY, San MA, Mehta PK, Dikalova AE, Garrido AM, et al. (2009) Mechanisms of vascular smooth muscle NADPH oxidase 1 (Noxl) contribution to injury-induced neointimal formation. Arterioscler. Thromb. Vasc. Biol. 29, $480-487$.

9. San MA, Lee MY, Williams HC, Mizuno K, Lassegue B, et al. (2008) Dual regulation of cofilin activity by LIM kinase and Slingshot-1L phosphatase controls platelet-derived growth factor-induced migration of human aortic smooth muscle cells. Circ. Res. 102, 432-438.

10. Umanskiy K, Robinson C, Cave C, Williams MA, Lentsch AB, et al. (2003) NADPH oxidase activation in fibronectin adherent human neutrophils: A potential role for betal integrin ligation. Surgery 134, 378-383.

11. Clempus RE, Sorescu D, Dikalova AE, Pounkova L, Jo P, et al. (2007) Nox4 is required for maintenance of the differentiated vascular smooth muscle cell phenotype. Arterioscler. Thromb. Vasc. Biol. 27, 42-48.

12. Diaz B, Shani G, Pass I, Anderson D, Quintavalle M, et al. (2009) Tks5dependent, nox-mediated generation of reactive oxygen species is necessary for invadopodia formation. Sci. Signal. 2, ra53.

13. Ushio-Fukai M, Tang Y, Fukai T, Dikalov SI, Ma Y, et al. (2002) Novel role of gp91(phox)-containing $\mathrm{NAD}(\mathrm{P}) \mathrm{H}$ oxidase in vascular endothelial growth factorinduced signaling and angiogenesis. Circ. Res. 91, 1160-1167.

14. Ikeda S, Ushio-Fukai M, Zuo L, Tojo T, Dikalov S, et al. (2005) Novel role of ARF6 in vascular endothelial growth factor-induced signaling and angiogenesis. Circ. Res. 96, 467-475.

15. Colavitti R, Pani G, Bedogni B, Anzevino R, Borrello S, et al. (2002) Reactive oxygen species as downstream mediators of angiogenic signaling by vascular endothelial growth factor receptor-2/KDR. J. Biol. Chem. 277, 3101-3108.

16. Bae YS, Lee JH, Choi SH, Kim S, Almazan F, et al. (2009) Macrophages generate reactive oxygen species in response to minimally oxidized low-density lipoprotein: toll-like receptor 4 and spleen tyrosine kinase-dependent activation of NADPH oxidase 2. Circ. Res. 104, 210-8, 21p.

17. Wells GM, Walmsley M, Ooi S, Tybulewicz V, and Ridley AJ (2004) Rac1deficient macrophages exhibit defects in cell spreading and membrane ruffling but not migration. J. Cell Sci. 117, 1259-1268.

18. Allen WE, Zicha D, Ridley AJ and Jones GE (1998) A role for Cdc42 in macrophage chemotaxis. J. Cell Biol. 141, 1147-1157.

19. Smith SD, Jaffer ZM, Chernoff J, and Ridley AJ (2008) PAK1-mediated activation of ERK1/2 regulates lamellipodial dynamics. J. Cell Sci. 121, 37293736 .

20. Sester DP, Trieu A, Brion K, Schroder K, Ravasi T, et al. (2005) LPS regulates a set of genes in primary murine macrophages by antagonising CSF-1 action. Immunobiology 210, 97-107.

21. Ridley AJ (2004) Pulling back to move forward. Cell 116, 357-358

22. Pankov R, Endo Y, Even-Ram S, Araki M, Clark K, et al. (2005) A Rac switch regulates random versus directionally persistent cell migration. J. Cell Biol 170, 793-802.

23. Merlot S and Firtel RA (2003) Leading the way: Directional sensing through phosphatidylinositol 3-kinase and other signaling pathways. J. Cell Sci. 116, 3471-3478.

24. Ward SG (2004) Do phosphoinositide 3-kinases direct lymphocyte navigation? Trends Immunol. 25, 67-74.

25. Leslie NR, Yang X, Downes CP, and Weijer GJ (2007) PtdIns(3,4,5)P(3)dependent and -independent roles for PTEN in the control of cell migration. Curr. Biol. 17, 115-125.

26. Procko E and McColl SR (2005) Leukocytes on the move with phosphoinositide 3-kinase and its downstream effectors. Bioessays 27, 153-163.

27. Leslie NR, Bennett D, Lindsay YE, Stewart H, Gray A, et al. (2003) Redox regulation of PI 3-kinase signalling via inactivation of PTEN. EMBO J. 22, $5501-5510$.

28. Denu JM and Tanner KG (1998) Specific and reversible inactivation of protein tyrosine phosphatases by hydrogen peroxide: evidence for a sulfenic acid intermediate and implications for redox regulation. Biochemistry 37, 56335642 .

29. Lee SR, Kwon KS, Kim SR, and Rhee SG (1998) Reversible inactivation of protein-tyrosine phosphatase $1 \mathrm{~B}$ in A431 cells stimulated with epidermal growth factor. J. Biol. Chem. 273, 15366-15372.
30. Ponting CP (1996) Novel domains in NADPH oxidase subunits, sorting nexins, and PtdIns 3-kinases: binding partners of SH3 domains? Protein Sci. 5, 23532357.

31. Ellson CD, Gobert-Gosse S, Anderson KE, Davidson K, Erdjument-Bromage $\mathrm{H}$, et al. (2001) PtdIns(3)P regulates the neutrophil oxidase complex by binding to the PX domain of p40(phox). Nat. Cell Biol. 3, 679-682.

32. Itoh RE, Kurokawa K, Ohba Y, Yoshizaki H, Mochizuki N, et al. (2002) Activation of rac and cdc42 video imaged by fluorescent resonance energy transfer-based single-molecule probes in the membrane of living cells. Mol. Cell Biol. 22, 6582-6591.

33. Srinivasan,S Wang,F Glavas,S Ott,A Hofmann,F etal. (2003). Rac and Cdc42 play distinct roles in regulating $\mathrm{PI}(3,4,5) \mathrm{P} 3$ and polarity during neutrophil chemotaxis. J. Cell Biol. 160, 375-385.

34. Etienne-Manneville S and Hall A (2002) Rho GTPases in cell biology. Nature 420, 629-635.

35. Diebold BA, Fowler B, Lu J, Dinauer MC, and Bokoch GM (2004) Antagonistic cross-talk between Rac and Cdc42 GTPases regulates generation of reactive oxygen species. J. Biol. Chem. 279, 28136-28142.

36. Kim JH, Na HJ, Kim CK, Kim JY, Ha KS, et al. (2008) The non-provitamin A carotenoid, lutein, inhibits NF-kappaB-dependent gene expression through redox-based regulation of the phosphatidylinositol 3-kinase/PTEN/Akt and NFkappaB-inducing kinase pathways: role of $\mathrm{H}(2) \mathrm{O}(2)$ in NF-kappaB activation. Free Radic. Biol. Med. 45, 885-896.

37. Touyz RM, Yao G, Quinn MT, Pagano PJ, and Schiffrin EL (2005) p47phox associates with the cytoskeleton through cortactin in human vascular smooth muscle cells: role in $\mathrm{NAD}(\mathrm{P}) \mathrm{H}$ oxidase regulation by angiotensin II. Arterioscler. Thromb. Vasc. Biol. 25, 512-518.

38. Morimatsu T, Kawagoshi A, Yoshida K, and Tamura M (1997) Actin enhances the activation of human neutrophil NADPH oxidase in a cell-free system. Biochem. Biophys. Res. Commun. 230, 206-210.

39. Bengtsson T, Orselius K, and Wettero J (2006) Role of the actin cytoskeleton during respiratory burst in chemoattractant-stimulated neutrophils. Cell Biol. Int. 30, 154-163.

40. Glading A, Uberall F, Keyse SM, Lauffenburger DA, and Wells A (2001) Membrane proximal ERK signaling is required for M-calpain activation downstream of epidermal growth factor receptor signaling. J. Biol Chem. 276, 23341-23348.

41. Fincham VJ, James M, Frame MC, and Winder SJ (2000) Active ERK/MAP kinase is targeted to newly forming cell-matrix adhesions by integrin engagement and v-Src. EMBO J. 19, 2911-2923.

42. Ishibe,S Joly,D Liu,Z.X., and Cantley,L.G. (2004) Paxillin serves as an ERKregulated scaffold for coordinating FAK and Rac activation in epithelial morphogenesis. Mol Cell 16, 257-267.

43. Schroder K, Helmcke I, Palfi K, Krause KH, Busse R, et al. (2007) Noxl mediates basic fibroblast growth factor-induced migration of vascular smooth muscle cells. Arterioscler. Thromb. Vasc. Biol. 27, 1736-1743.

44. Paik YH, Iwaisako K, Seki E, Inokuchi S, Schnabl B, et al. (2011) The nicotinamide adenine dinucleotide phosphate oxidase (NOX) homologues NOX1 and NOX2/gp91(phox) mediate hepatic fibrosis in mice. Hepatology 53, 1730-1741.

45. Cui W, Matsuno K, Iwata K, Ibi M, Matsumoto M, et al (2011) NOX1/ nicotinamide adenine dinucleotide phosphate, reduced form (NADPH) oxidase promotes proliferation of stellate cells and aggravates liver fibrosis induced by bile duct ligation. Hepatology 54, 949-958.

46. Bendall JK, Cave AC, Heymes C, Gall N, and Shah AM (2002) Pivotal role of a gp91(phox)-containing NADPH oxidase in angiotensin II-induced cardiac hypertrophy in mice. Circulation 105, 293-296.

47. Johar S, Cave AC, Narayanapanicker A, Grieve DJ, and Shah AM (2006) Aldosterone mediates angiotensin II-induced interstitial cardiac fibrosis via a Nox2-containing NADPH oxidase. FASEB J. 20, 1546-1548.

48. Byrne JA, Grieve DJ, Bendall JK, Li JM, Gove C, et al. (2003) Contrasting roles of NADPH oxidase isoforms in pressure-overload versus angiotensin II-induced cardiac hypertrophy. Circ. Res. 93, 802-805.

49. Bondi CD, Manickam N, Lee DY, Block K, Gorin Y, et al. (2010) NAD(P)H oxidase mediates TGF-betal-induced activation of kidney myofibroblasts. J. Am. Soc. Nephrol. 21, 93-102.

50. Zhong J, Guo D, Chen CB, Wang W, Schuster M, et al. (2011) Prevention of angiotensin II-mediated renal oxidative stress, inflammation, and fibrosis by angiotensin-converting enzyme 2. Hypertension 57, 314-322.

51. Liu J, Yang F, Yang XP, Jankowski M, and Pagano PJ (2003) NAD(P)H oxidase mediates angiotensin II-induced vascular macrophage infiltration and medial hypertrophy. Arterioscler. Thromb. Vasc. Biol 23, 776-782.

52. Vendrov AE, Hakim ZS, Madamanchi NR, Rojas M, Madamanchi C, et al. (2007) Atherosclerosis is attenuated by limiting superoxide generation in both macrophages and vessel wall cells. Arterioscler. Thromb. Vasc. Biol 27, 2714 2721 .

53. Thomas M, Gavrila D, McCormick ML, Miller FJ, Daugherty A, et al. (2006) Deletion of p47phox attenuates angiotensin II-induced abdominal aortic aneurysm formation in apolipoprotein E-deficient mice. Circulation 114, 404 413. 